\title{
The Arabidopsis At1g30680 gene encodes a homologue to the phage T7 gp4 protein that has both DNA primase and DNA helicase activities
}

\author{
Joann Diray-Arce ${ }^{\dagger}$, Bin Liu ${ }^{\dagger}$, John D Cupp, Travis Hunt and Brent L Nielsen*
}

\begin{abstract}
Background: The Arabidopsis thaliana genome encodes a homologue of the full-length bacteriophage T7 gp4 protein, which is also homologous to the eukaryotic Twinkle protein. While the phage protein has both DNA primase and DNA helicase activities, in animal cells Twinkle is localized to mitochondria and has only DNA helicase activity due to sequence changes in the DNA primase domain. However, Arabidopsis and other plant Twinkle homologues retain sequence homology for both functional domains of the phage protein. The Arabidopsis Twinkle homologue has been shown by others to be dual targeted to mitochondria and chloroplasts.

Results: To determine the functional activity of the Arabidopsis protein we obtained the gene for the full-length Arabidopsis protein and expressed it in bacteria. The purified protein was shown to have both DNA primase and DNA helicase activities. Western blot and qRT-PCR analysis indicated that the Arabidopsis gene is expressed most abundantly in young leaves and shoot apex tissue, as expected if this protein plays a role in organelle DNA replication. This expression is closely correlated with the expression of organelle-localized DNA polymerase in the same tissues. Homologues from other plant species show close similarity by phylogenetic analysis.
\end{abstract}

Conclusions: The results presented here indicate that the Arabidopsis phage $\mathrm{T7} \mathrm{gp} 4 / \mathrm{T}$ winkle homologue has both DNA primase and DNA helicase activities and may provide these functions for organelle DNA replication.

Keywords: DNA primase, DNA helicase, bacteriophage T7 gp4, Twinkle, Organelle DNA replication

\section{Background}

DNA replication involves the coordinated activity of several enzymes and proteins. These enzymes assist with the unwinding, separation, and copying of double stranded DNA to produce new identical DNA copies [1]. DNA helicase translocates unidirectionally along one strand of the nucleic acid to facilitate replication initiation. The helicase utilizes ATP hydrolysis to separate the DNA double helix into individual strands [2,3]. DNA primase catalyzes the formation of short RNA oligonucleotides used as primers to begin DNA synthesis [4]. DNA polymerase uses the primers and extends the 3 ' end of the nucleotide chain by adding nucleotides matched to the template strand [1].

\footnotetext{
* Correspondence: brentnielsen@byu.edu

${ }^{\dagger}$ Equal contributors

Dept. of Microbiology \& Molecular Biology, 775 WIDB, Brigham Young University, Provo, Utah 84602, USA
}

(c) 2013 Diray-Arce et al.; licensee BioMed Central Ltd. This is an Open Access article distributed under the terms of the Creative Commons Attribution License (http://creativecommons.org/licenses/by/2.0), which permits unrestricted use, distribution, and reproduction in any medium, provided the original work is properly cited.
Individual genes are usually responsible for encoding each replication enzyme activity. However, bacteriophage T7 gene 4 protein (T7 gp4) and similar proteins from T3, P4 and other phages [4] encode a single protein with both DNA helicase and DNA primase domains. T7 phage has two forms of gp4 protein that are both required for phage genome replication. The longer form has two zinc motifs and has both DNA primase and helicase activity while the shorter one retains only DNA helicase activity [5].

Most eukaryotic organisms have a homologue of the T7 gp4 protein that has been named Twinkle (T7 gp4-like protein with intramitochondrial nucleoid localization). This protein shares close sequence similarity with the bacteriophage T7 gp4 primase-helicase protein [6,7]. Twinkle is a hexameric DNA helicase at the mitochondrial DNA replication fork which unwinds sections of double-stranded DNA [8,9]. The Twinkle homologue lacks DNA primase activity in higher 
eukaryotes but is suggested to have this activity in Plasmodium species $[6,10]$ and Arabidopsis thaliana and other plants $[11,12]$. This protein is assumed to play a key role in mitochondrial DNA (mtDNA) replication as it localizes in the mitochondrial nucleoid and matrix. In maize, Twinkle has also been found associated with the chloroplast nucleoid [13], suggesting that this protein may function in both mitochondria and chloroplasts.

Mutations in Twinkle result in mitochondrial-associated diseases in humans $[6,14]$ and mice $[15,16]$. In humans, coding region mutations in this gene have been linked with autosomal dominant progressive external ophthalmoplegia (adPEO) and are often associated with multiple mtDNA deletions, suggesting a role in mtDNA replication [6]. In mice, Twinkle expression reduction by RNAi resulted in a rapid drop in mtDNA copy number $[6,17]$ while overexpression of the protein led to increases in mtDNA copy number in muscle and heart tissue $[15,18]$.

When the amino acid sequences of Twinkle homologues from a wide variety of eukaryotic species are compared, high homology in the conserved Walker motifs for the DNA helicase domain of the protein has been observed, as summarized in two review papers $[4,5]$. Critical differences were observed in the primase domain of Twinkle in some model organisms when compared to the N-terminal end of the T7 gp4 protein [19]. The location of the (nonfunctional) primase domain in human Twinkle is at the $\mathrm{N}$-terminal portion of the protein, the same as in phage T7 gp4 and in DNAG-like primases in bacteria and phage $[4,11]$. But unlike T7 gp4, the Nterminal domain of human Twinkle lacks several motifs required for primer synthesis in $\mathrm{T} 7 \mathrm{gp} 4$, thus leading to the prediction that the Twinkle $\mathrm{N}$-terminal region is generally inactive in humans and metazoa in general [5]. The T7 gp4 protein contains a beta sheet structure and cysteine residues forming two zinc fingers [7] in Motif 1. The N-terminal end of the primase domain of T7 gp4 contains a zinc finger motif but Twinkle in most metazoan species lacks the zinc-binding domain necessary for DNA and amino acid binding for polymerization of the primer [5]. Also, human Twinkle does not contain the conserved cysteine residues of the zinc-finger motif critical for DNA binding and primase activity [20]. The zinc finger motif in the primase domain synthesizes pppAC oligonucleotide primers important for the initial step of sequence-specific primer synthesis at the sequence $5^{\prime}$-GTC-3' [21]. The Twinkle protein from Arabidopsis thaliana contains the conserved sequence elements and is predicted to have both DNA primase and DNA helicase activities.

The Arabidopsis genome contains two homologues of the bacteriophage T7 gp4 protein. The first (At1g30680) shares homology with the conserved motifs of the DNA primase and DNA helicase domains [5]. The coding sequence predicts a protein of about $80 \mathrm{kDa}$, which is larger than the full-length $63,000 \mathrm{kDa}$ T7 gp4 protein but similar to the sizes of Twinkle homologues reported in eukaryotes. The second Arabidopsis homologue is truncated, sharing the $\mathrm{N}$-terminal primase domain but entirely lacking the C-terminal helicase domain, with a predicted size of $\sim 38 \mathrm{kDa}$ (At1g30660). The truncated gene will be designated as a primase homologue, while the full-length gene will be designated as a Twinkle homologue in this paper.

We show here that the Arabidopsis T7 gp4 homologue has both DNA primase and DNA helicase activities, the first such report from a higher eukaryote. The gene for this protein is highly expressed in rapidly growing plant tissues and is correlated with organelle DNA polymerase gene expression.

\section{Results}

\section{Expression of the Arabidopsis protein in E. coli and} demonstration of DNA primase activity

The full-length cDNA for the Arabidopsis Twinkle gene was obtained and cloned into a bacterial expression vector to produce protein for enzymatic activity assays. The purified protein showed a predominant band of the proper size by gel staining (Figure 1A). Its identity as the expressed protein was confirmed by western blot analysis using an antibody against a synthetic peptide from the Arabidopsis protein sequence (Figure 1B). The recombinant protein product is smaller $(\sim 74 \mathrm{kDa})$ than the full-length coding region of the Twinkle homologue since it lacks the N-terminal organelle targeting sequence. The purified protein was used for an in vitro assay for DNA primase activity. Gel analysis of the reaction products indicates that the protein is capable of producing RNA primers of $\sim 9-18$ bases from a singlestranded DNA template (Figure 2). Stronger intensity of the primers of 9 and 14 bases was consistently observed (close-up shown in Figure 2A), and are similar in size to products reported for other DNA primases [22]. The primers were capable of being extended by DNA polymerase into high molecular weight DNA (Figure 2B), which is a fundamental property of a DNA primase that generates primers for DNA replication. The primer bands are absent in the control lanes (protein from bacteria with the empty vector lacking the Arabidopsis gene), indicating that this activity is not due to bacterial DNA primase contamination of the purified recombinant protein. This provides clear evidence for the function of the Arabidopsis Twinkle homologue as an active DNA primase, the first such report in a higher eukaryote. 


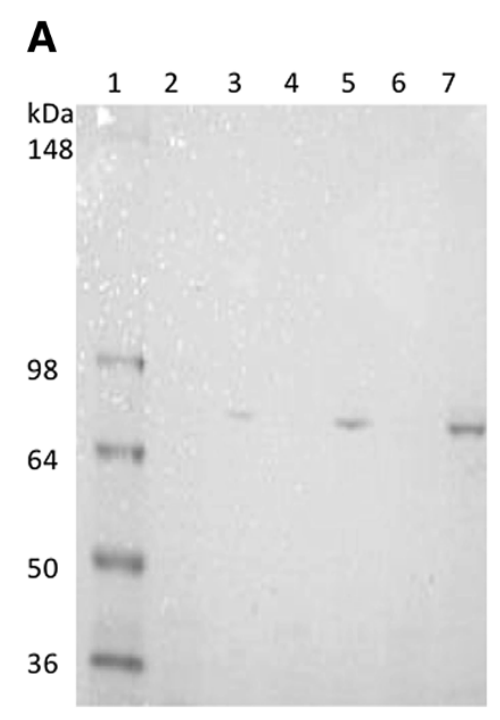

B

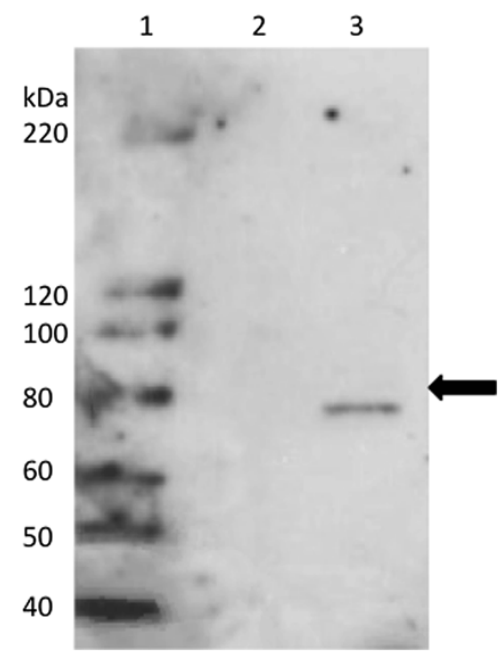

Figure 1 Purification of the recombinant protein. Panel A shows the Coomassie Blue-stained gel, with increasing amounts of the purified recombinant (lanes 3, 5 and 7) and control (lanes 2, 4 and 6) protein, from left to right. Lane 1, protein molecular weight markers (Invitrogen SeeBlue 2 markers). Lanes 2 and 3, 0.195 ng; lanes 4 and 5, 0.39 ng; lanes 6 and 7, 0.585 ng. Panel B shows a western blot of the purified protein using antibody against the Arabidopsis Twinkle homologue. Lane 1 contains molecular weight markers (Invitrogen Magic Markers). Lane 2, control protein; lane 3, $0.5 \mathrm{ng}$ purified recombinant protein. The arrow at the right indicates $80 \mathrm{kDa}$, the length of the full-length Arabidopsis gene product. The recombinant protein is slightly smaller $(\sim 74 \mathrm{kDa})$ as it lacks the $\mathrm{N}$-terminal localization sequence.

\section{DNA helicase activity of the Arabidopsis Twinkle homologue protein}

The purified recombinant protein was also assayed for DNA helicase activity. The results indicate that the protein indeed has ATP-dependent DNA helicase activity as predicted (Figure 3). The control protein preparation (vector with no insert) lacked DNA helicase activity in the presence or absence of ATP (Figure 3 lanes 5 and 6). The activity is similar to the DNA unwinding activity we previously detected in soybean mitochondrial extracts [23]. The results from the biochemical assays indicate that the Arabidopsis Twinkle homologue has both DNA primase and helicase activities, similar to the phage T7 gp4 protein.

\section{Western blot analysis of Arabidopsis Twinkle homologue expression}

Western blot analysis of Twinkle protein expression levels in different Arabidopsis tissues shows that the protein is most abundant in meristem and young leaf tissue and nearly undetectable in mature leaves (Figure 4A). Total rosette leaf tissue from plants was collected at weekly intervals and total protein was recovered from each sample for western blot analysis. The results show relatively high levels of the Twinkle protein in weeks 1-3 of growth, with a subsequent rapid drop in levels until the protein is nearly undetectable after week 5 (Figure 4B). These results are compatible with those reported from the different tissues (Figure 4A). Western blot analysis indicated the presence of Twinkle in isolated mitochondria and chloroplasts of Arabidopsis (data not shown).

\section{Analysis of Arabidopsis Twinkle homologue expression in different tissues by qRT-PCR}

Quantitative reverse transcriptase PCR analysis of cDNA generated from different tissues indicate that the Arabidopsis Twinkle gene is expressed at the highest level in the shoot apex (Figure 5), as expected if the Twinkle protein plays a role in organelle DNA replication in rapidly growing tissues. Twinkle is also expressed at relatively high levels in other developing tissues, especially cotyledons and different parts of flowers including sepals, pistils and the inflorescence (Figure 5). Interestingly, expression levels of Twinkle are very similar to expression levels of DNA Pol gamma I (Figure 5), a dual-targeted DNA polymerase that has been shown to play a role in plant organelle DNA replication and repair [24]. The expression of DNA Pol gamma II is also generally highest in the same tissues that have high Twinkle expression (Figure 5). The similar levels of expression of Twinkle and the organelle-localized DNA polymerases [25] suggest that Twinkle may play a role in replication of organelle DNA.

\section{Analysis of Twinkle DNA and protein sequences}

Two separate research groups have reported on the comparison of the amino acid sequences of Twinkle 


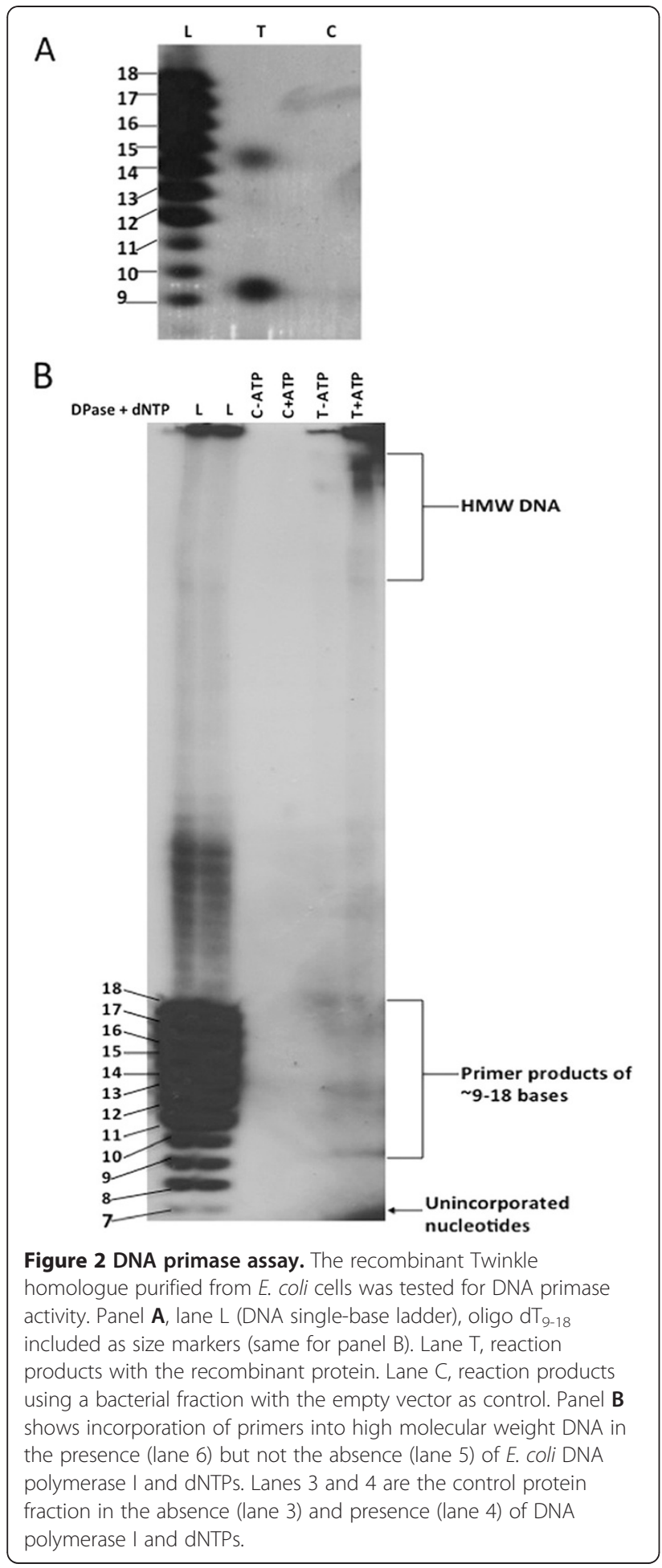

homologues from a wide variety of eukaryotic species, and have shown that there is high homology in the conserved Walker motifs for the C-terminal DNA helicase domain of the Arabidopsis protein [4,5]. The human, Drosophila and C. elegans Twinkle homologues have DNA helicase activity but lack DNA primase activity

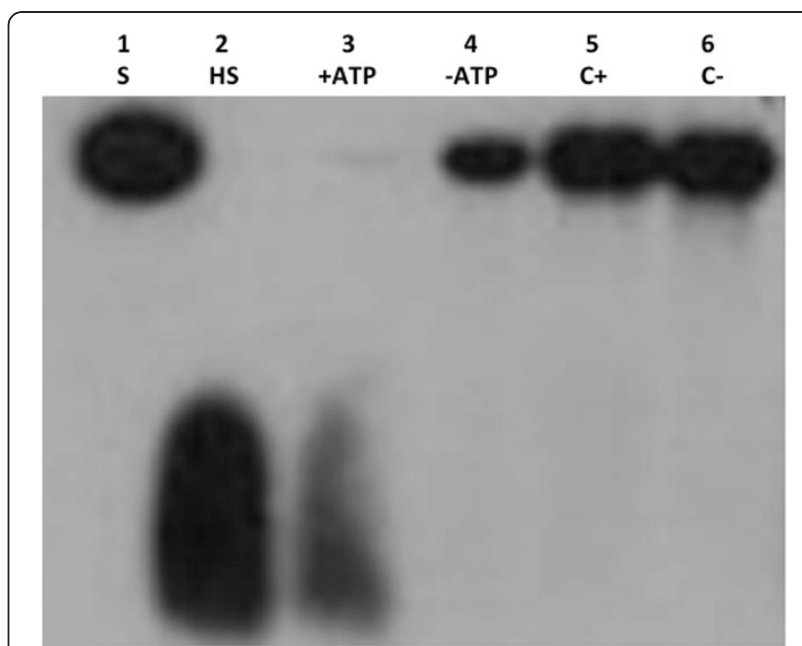

Figure 3 DNA helicase assay. The recombinant Twinkle homologue purified from E. coli cells was tested for DNA helicase activity as described in the text. Lane 1 is the control substrate (S). Lane 2 is the heated control (HS), showing separation of the short labeled oligo from the substrate, which runs in this gel as a leading band with a diffuse smear; lane $3(T+A T P)$, reaction using the purified recombinant protein with ATP; lane 4 (T-ATP), same reaction without ATP, lane 5 (C+ATP), control protein from $E$. coli cells lacking the expression construct with ATP, lane 6 (C-ATP), same reaction but without ATP.

$[4,5]$. Upon close examination of the amino acid sequence encoding the primase domain at the $\mathrm{N}$-terminal end of the protein in the plant and animal proteins, some critical differences are apparent. Two zinc fingers formed by cysteine residues in Motif 1 are present in the T7 gp4 protein [7] and in homologues from most eukaryotes, but the four cysteines that form the zinc fingers are absent in metazoans, including humans $[4,5]$. Analysis of the amino acid sequence alignment of the Twinkle homologues against the T7 gp4 protein shows that only the Arabidopsis and other plant Twinkle homologues share all highly conserved elements with the T7 gp4 protein [5]. Additional important differences are observed in other conserved motifs within the primase region of the protein in humans, Drosophila and $C$. elegans, while the sequences from a number of lower eukaryotes share the conserved elements with $\mathrm{T} 7 \mathrm{gp} 4$ protein [5]. In particular, the human homologue lacks both zinc finger domains in Motif 1, and the human and Drosophila sequences lack the highly conserved residues found in Motif IV and Motif V.

The Arabidopsis thaliana Twinkle protein contains the conserved sequence elements and is predicted to have both DNA primase and DNA helicase activities [4]. While the previous analysis of the amino acid sequences of these proteins identified critical differences at some conserved sites in the primase domain region of the protein in metazoa, including the absence of the cysteine residues needed to form the zinc fingers $[4,5]$, we 


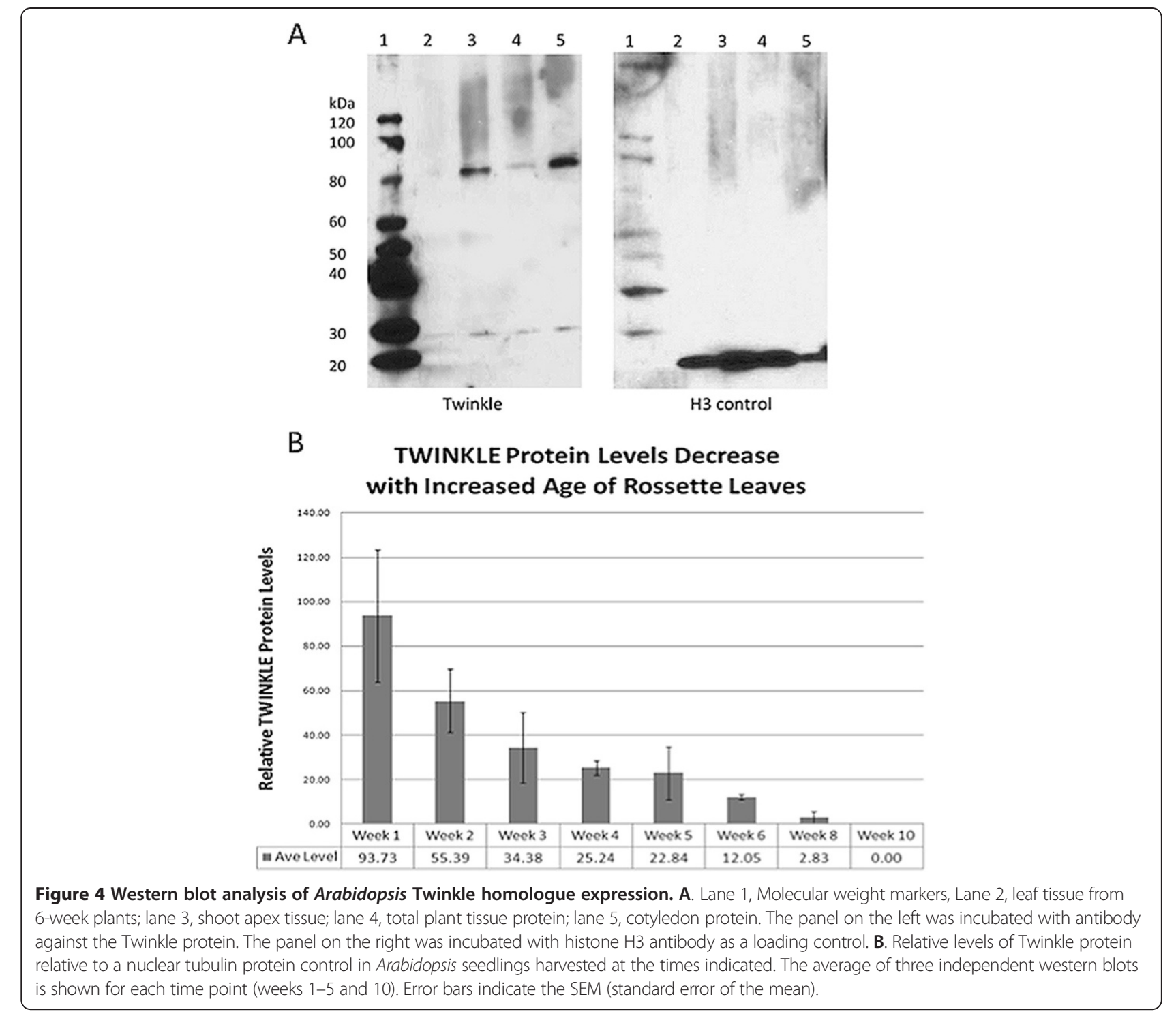

wanted to know if these changes were due to minor mutations in the sequence. However, DNA sequence analysis indicates that the differences in amino acid sequence of the homologues in human and Drosophila are not due to single base changes but are due to more significant alterations in the DNA sequence (Figure 6). The base sequence differences that are present in the Arabidopsis Twinkle primase domain as compared to the T7 gp4 protein mostly occur in the third position of the codons and do not alter the amino acid sequence.

Phylogenetic analysis of amino acid sequences of Twinkle homologues from several plants and other species shows that the Arabidopsis and plant homologues are closely clustered and are most similar to the bacteriophage T7 gp4 protein (Figure 7). The relationship between Twinkle proteins is supported by maximum likelihood phylogenetic analysis of taxonomic samples of
Twinkle homologues. This suggests that the Twinkle homologues from humans and other animals are most distantly related to the T7 gp4 protein, supporting the observations from direct DNA and amino acid sequence alignments.

\section{Discussion}

Twinkle has been shown to be the replicative DNA helicase in mitochondria of eukaryotic cells, and mutations that abolish expression of this gene are lethal in animal cells $[6,14,15,26]$. Twinkle is a homologue of the bacteriophage T7 gp4 protein, which has both DNA primase and DNA helicase activities and contains the highly characterized TOPRIM domain that is conserved in DNA primases, topoisomerases and OLD family nucleases [4]. However, until the present work no Twinkle 


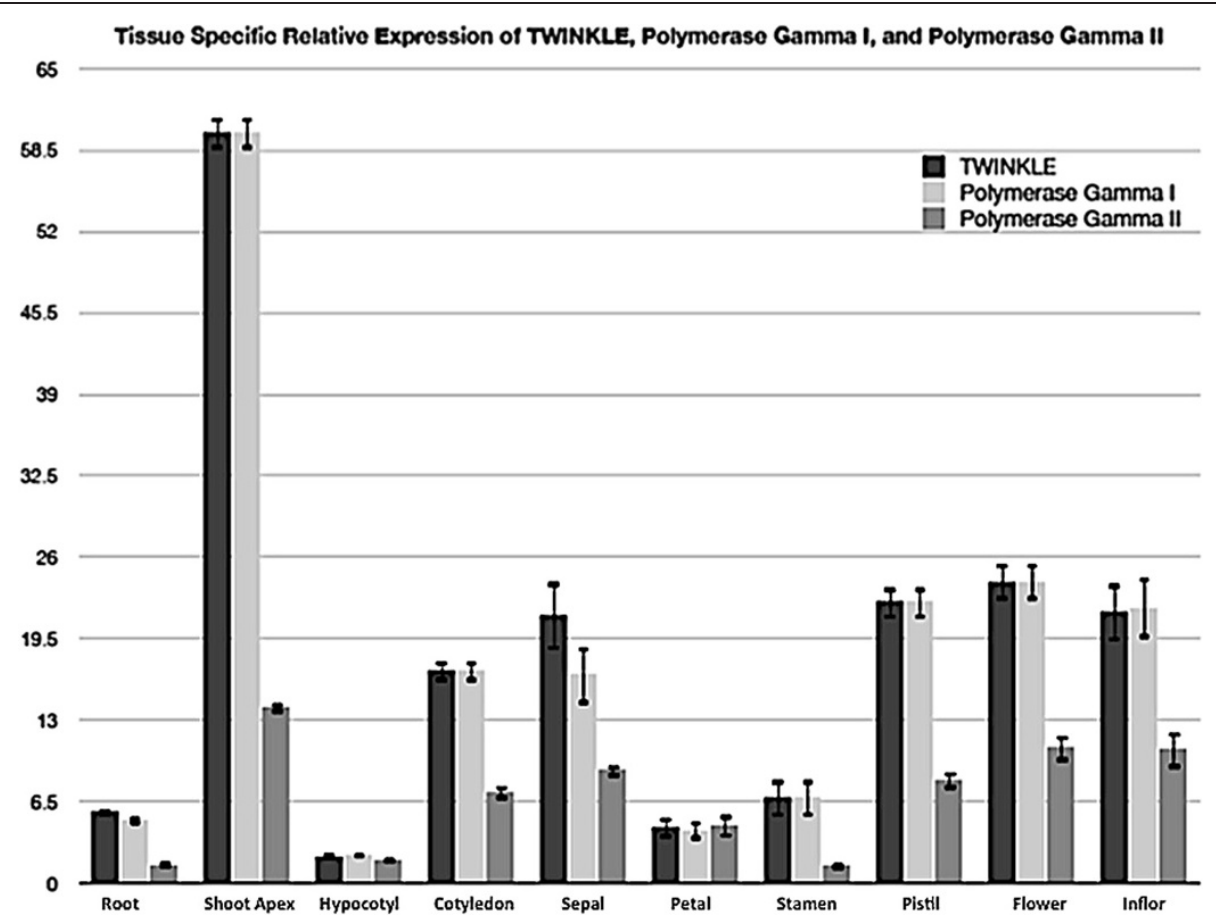

Figure 5 RT-qPCR analysis of the Arabidopsis Twinkle homologue gene expression relative to organellar localized DNA polymerases in various tissues. The relative abundance of Twinkle and the two organellar DNA polymerases (Polymerase gamma I and Polymerase gamma II) is shown. Expression varied among selected organs with highest expression in the shoot apex. The relative expression of Twinkle follows the expression levels of DNA polymerase gamma I. Error bars indicate SEM of three replicates. The $Y$ axis indicates relative expression ( $\log _{2}$ ) normalized to nuclear actin gene expression. Inflor, inflorescence.

homologue from a higher eukaryote has been shown to have DNA primase activity. Shutt and Gray have analyzed the sequence of Twinkle homologues from several eukaryote species and have proposed that in addition to being the DNA helicase, Twinkle may also serve as the mitochondrial DNA primase in most eukaryotes except metazoa [5]. As far as we know our present report is the first to show that the Twinkle homologue in a plant species (Arabidopsis) has both DNA primase and DNA helicase activities. Other than the truncated primase homologue already mentioned (At1g30660; but there is no information available about whether this protein is functional) no other bacterial or phage-type DNA primase homologues have been found in the Arabidopsis genome sequence.

Sequence analysis provides an explanation of why the plant homologue has both activities while the animal homologues lack DNA primase activity (Figure 6). The absence of primase activity in human Twinkle is likely due to the lack of the zinc finger motifs formed by 4 cysteine residues near the $\mathrm{N}$-terminal end of the protein, as well as other amino acid sequence differences at conserved sequences in the primase domain of the protein which have been shown to be responsible for the primase activity (Figure 6) [4]. Sequence variation occurs in other metazoan species, and while some have the zinc fingers, they have differences at other conserved motifs. The Arabidopsis homologue, in contrast, retains all conserved motifs [4]. Phylogenetic analysis further supports these findings, indicating that the plant Twinkle homologues are most closely related to the $\mathrm{T} 7 \mathrm{gp} 4$ protein, while the animal homologues are quite distantly related. These results suggest that the bifunctional $\mathrm{T} 7 \mathrm{gp} 4$ homologue may be conserved in higher plants.

The Arabidopsis Twinkle protein may function both in mitochondria and chloroplasts, as this protein has been shown to be dual-targeted to both organelles $[27,28]$. These reports are based on the analysis of predicted Nterminal targeting sequences of a number of nuclearencoded Arabidopsis proteins fused with the GFP coding region. However, it has been shown that targeting of fusion proteins can be affected by the context of the $\mathrm{N}$-terminal sequence with the GFP sequence $[28,29]$. A recent report on the maize plastid proteome has shown the presence of Twinkle in the chloroplast nucleoid [13].

Mitochondrial genomes range widely in size, from about $16.5 \mathrm{kbp}$ in vertebrates and invertebrates, to $70-100 \mathrm{kbp}$ in yeast and 200-2000 kbp in plants. The replication of animal mtDNA has been characterized in great detail, and in the original model each strand of the duplex DNA replicates at a different time, with the initial replication primed by a short transcript synthesized by the 


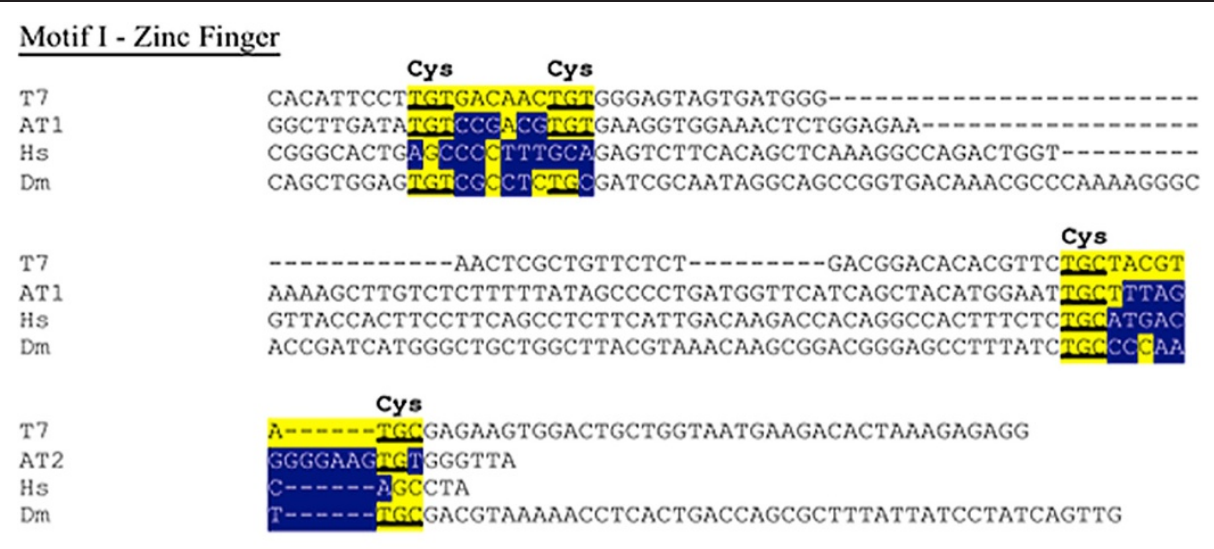

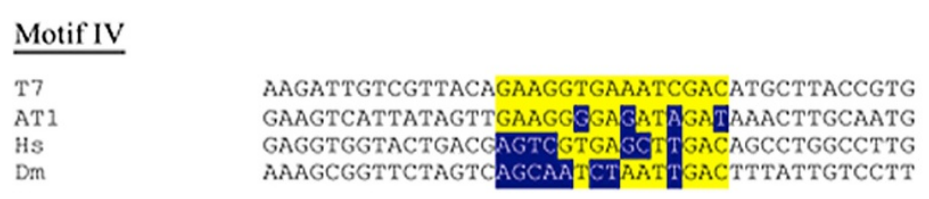

Motif V
T7
AT1
Hs
Dm

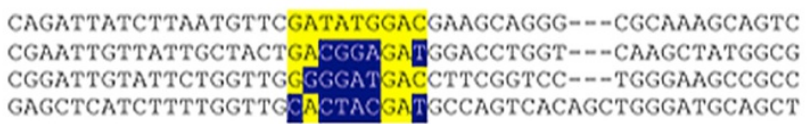

Figure 6 A. DNA sequence alignments of some Twinkle primase domain conserved regions to show the extent of changes between different organisms. The DNA sequences for the Twinkle protein from T7, Arabidopsis (At1g30680), human (Hs) and Drosophila (Dm) are shown for the conserved motifs I, IV and V. The locations of the cysteine residues in Motif I are indicated above the sequence while the corresponding codon sequence is underlined in the DNA sequence. The central conserved elements of each motif are shaded yellow. Base differences from the T7 gp4 sequence are shaded dark blue with white lettering.

mitochondrial RNA polymerase [30]. The second strand replicates only when it becomes single stranded by progression of the first strand, allowing formation of a characteristic structure to facilitate replication initiation of this strand. In yeast and plants, mtDNA replication appears to be more complex, and may involve a recombinationdependent replication mechanism [23,31-34]. In this case DNA priming may not be required if invading strands provide the priming function for DNA synthesis. However, even in phage systems that replicate by a recombination mechanism a DNA primase is still required for priming synthesis at lagging strands during some phases of DNA replication [4].

A distinct mtDNA primase activity has been reported in some animal and protist cells and mtDNA primase activity has been reported in human cells, but no distinct human protein with this activity has yet been identified. It has been suggested that the DNA primase in animal

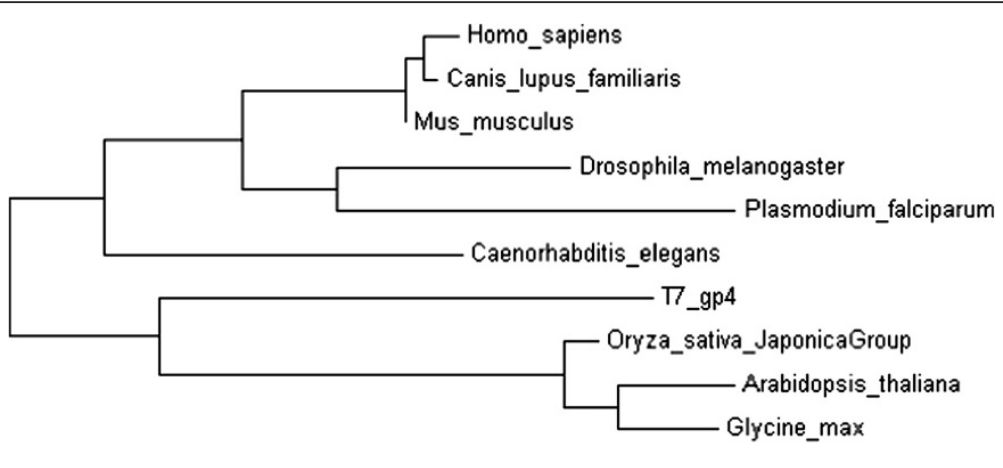

0.2

Figure 7 Phylogenetic analysis of the T7 gp4 protein, plant homologues, and selected eukaryotic Twinkle protein homologues. Molecular phylogenetic analysis was performed using the maximum likelihood method. The scale bar indicates the number of substitutions per site. 
cells is tightly associated with the mtDNA $(\gamma)$ polymerase, and is thus difficult to isolate separately [35]. In a trypanosome a mtDNA primase of $70 \mathrm{kDa}$ has been reported [36], while in yeast a mtDNA primase of $67 \mathrm{kDa}$ has been characterized [37], which are both close to the size of T7 gp4 and Twinkle. Our understanding of animal mtDNA replication is complicated by reports of strand-coupled bidirectional replication from a single replication origin, which by its nature should require a DNA primase to synthesize primers for the lagging strand $[30,38,39]$. It is unclear whether a separate mtDNA primase is present or required in species (including human) with highly compact mitochondrial genomes [40]. Recently it has been shown that in vitro, human mitochondrial RNA polymerase is responsible for priming lagging strand mtDNA synthesis. It may be possible that priming of replication of the small animal mitochondrial genome is provided by short transcripts synthesized by the mitochondrial RNA polymerase [40,41].

A DNA primase has been purified and characterized from pea chloroplasts [42], and primers synthesized by that preparation are similar in size to primers synthesized by the purified Arabidopsis Twinkle homologue. The pea enzyme is larger $(\sim 90 \mathrm{kDa})$ than the Arabidopsis Twinkle homologue, but it was not characterized for DNA helicase activity. CtDNA replication involves multiple replication origins and bidirectional DNA synthesis [42,43], which would require DNA primase activity for lagging strand synthesis.

Organelle DNA replication appears to be different in plants (as compared to animals), which have very large and complex mitochondrial genomes and likely require multiple sites of lagging strand DNA synthesis. The role of recombination-mediated replication [33,34] may reduce the need for primase-synthesized primers for organelle DNA replication, as an invading DNA strand could provide the $3^{\prime}$ ends for DNA synthesis. However, even in this case it is likely that organelle DNA primase is required in plants. Bacteriophage T4 replicates by multiple mechanisms, including recombinationdependent replication, and requires a DNA primase. The observations that the Arabidopsis Twinkle protein is expressed at highest levels in the shoot apex and other developing tissues including young leaves provides strong support for a role of the Twinkle homologue in plant organelle DNA replication, similar to its role in other species $[4,5]$.

Mutations in human Twinkle have been shown to lead to a drastic reduction in mtDNA copy number and disease [17]. RNAi-mediated reduction of Twinkle expression in cultured human cells was found to lead to a rapid drop in mtDNA copy number, while overexpression of Twinkle in mouse tissue was associated with an increase in mtDNA copy number $[15,26]$. In each of these cases the effect has been associated with the DNA helicase activity of the protein. We showed that this single protein from Arabidopsis has both DNA primase and DNA helicase activities in vitro, the same activity as the bacteriophage $\mathrm{T} 7 \mathrm{gp} 4$ protein.

\section{Conclusion}

The Arabidopsis homologue of the bacteriophage T7 gp4 protein has been shown to have both DNA primase and DNA helicase activities similar to the phage protein. It is expressed at highest levels in actively growing tissues, suggesting that it could play a role in organelle DNA replication. Two DNA polymerases have been identified in plants, and both have been reported to be dual targeted to mitochondria and chloroplasts [28,44]. It is likely that this Arabidopsis phage T7 gp4 homologue functions along with one or both of these DNA polymerases to accomplish organelle DNA replication. Even if the mtDNA replicates by a recombination-dependent mechanism as suggested by some [23,33,34], DNA priming may be required for lagging-strand DNA replication. This Arabidopsis protein may also play a role in control of plant mtDNA (and possibly also ctDNA) copy number as observed in animals [5,17], but this determination will require additional experiments, which will be the subject of future work in our lab.

\section{Methods}

Identification of an Arabidopsis Twinkle homologue

A full-length Twinkle homologue was identified in the Arabidopsis thaliana genome (At1g30680, protein molecular weight of $80,401.9 \mathrm{Da})$. A second, truncated homologue is also present (At1g30660, molecular weight of $37,806.9 \mathrm{Da}$ ) near the first gene, but contains only the primase domain of the protein and ends near the linker region [45] joining the primase and helicase domains. Only the full-length gene (At1g30680) was examined in this study.

\section{Recombinant expression of the Arabidopsis Twinkle homologue}

The full-length cDNA for At1g30680 was obtained from Riken (Japan). The full-length coding region for this gene predicts a polypeptide of 709 amino acids, and the MitoProt program [46] predicts the cleavage site after amino acid 91, which is prior to the conserved elements including the zinc fingers in the DNA primase domain of the protein. We generated a construct of the entire conserved coding region of the gene but lacking the DNA sequence for the N-terminal 91 amino acids in the pEXP5-NT/TOPO expression vector (Invitrogen). The construct was then transformed into the E. coli BL21 strain (Invitrogen). A total volume of $500 \mathrm{ml}$ of LB was 
used to grow the bacteria. After it reached O.D.600 0.4-0.6, IPTG was added to the medium to a final concentration of $0.5 \mathrm{mM}$ to induce the expression of the targeted protein. The cells were grown at $30^{\circ} \mathrm{C}$ for an additional $4 \mathrm{hr}$ and harvested by centrifugation. A control strain containing an empty vector lacking the gene insert was grown under identical conditions. The recombinant protein and control sample were purified under identical conditions using ProBond Nickel-chelating resin (Invitrogen). Native conditions were used and the purification was performed as described in the manual. Protein purity was analyzed by gel electrophoresis and western blot analysis.

\section{DNA primase activity assay}

DNA primase activity of the recombinant protein was detected using a previously published procedure [42] using single-stranded M13 DNA as template. A control bacterial fraction was included to eliminate the possibility that bacterial DNA primase was present in the recombinant protein fraction. Single-stranded M13 DNA was incubated with $0.5 \mathrm{ng}$ of the ProBond-purified recombinant or control protein fraction in the presence of rNTPs including $\alpha^{32} \mathrm{P}$-ATP (MP Biomedical). The reaction products were separated in a $20 \%$ denaturing polyacrylamide gel (6\% urea in $1 \mathrm{X} \mathrm{TBE}$ ). End-labeled oligo $(\mathrm{dT})_{12-18}$ was used as size markers. After electrophoresis the gel was dried and exposed to X-ray film.

\section{DNA helicase activity assay}

DNA helicase activity of the ProBond-purified recombinant protein was assayed according to the procedure of Song [47].The substrate was prepared by annealing (heating for $5 \mathrm{~min}$ to $65^{\circ} \mathrm{C}$ in $40 \mathrm{mM}$ Tris- $\mathrm{HCl}, \mathrm{pH} 7.8$, $50 \mathrm{mM} \mathrm{NaCl}$ and slowly cooling to room temperature for 20-30 min) single-stranded M13 circular DNA with a complementary oligonucleotide ( $5^{\prime}$ GTAAAACGACG GCCAGT $3^{\prime}$ ) labeled at the $5^{\prime}$ end using T4 polynucleotide kinase (New England Biolabs) and $\gamma^{32}$ P-ATP (MP Biomedical). The substrate was incubated with $0.5 \mathrm{ng}$ of the recombinant protein in reaction buffer $(10 \mathrm{mM}$ Tris- $\mathrm{HCl}, \mathrm{pH} 8.0,8 \mathrm{mM} \mathrm{MgCl}, 1 \mathrm{mM}$ dithiothreitol, $5 \mathrm{mM}$ ATP, $1 \mathrm{ng}{ }^{32}$ P-labeled helicase substrate) for $30 \mathrm{~min}$, after which the reaction was terminated by adding EDTA to $2 \mathrm{mM}$, and the reaction products were separated by electrophoresis through a native TBE $6 \%$ polyacrylamide gel. The same bacterial protein control was included. The gel was then dried and exposed to X-ray film.

\section{Western blot analysis of Twinkle homologue expression in different tissues}

Protein fractions were prepared from different tissues of Arabidopsis thaliana by grinding in liquid nitrogen and suspending in $1 \mathrm{X}$ SDS-loading buffer. The proteins were heated to $95^{\circ} \mathrm{C}$ for $5 \mathrm{~min}$ and separated by electrophoresis in $8-20 \%$ SDS-PAGE gels. Proteins were transferred to PVDF membrane and after blocking in 5\% skim milk the membrane was incubated with antibody that had been raised in rabbit (by Sigma-Genosys) against a synthetic peptide from a unique region of the Twinkle protein (KASRIVIATDGDGPG). This sequence is shared in both the full-length and truncated Arabidopsis genes (At1g30680 and At1g30660). The sequence of the peptide antigen was compared to the entire Arabidopsis proteome to ensure it does not share homology with any other protein besides the Twinkle homologues (NCBI-Blast). A control blot against the histone $\mathrm{H} 3$ protein was performed for normalization of signal strength. Bound antibody was detected using the Pierce Supersignal Western Chemiluminescence kit followed by exposure to X-ray film.

For time course analysis, total leaf tissue was extracted from Arabidopsis plants at weekly intervals starting at 1 week of age. The tissue was flash frozen in liquid nitrogen and stored at $-80^{\circ} \mathrm{C}$. Total protein was extracted from $50 \mathrm{mg}$ of crushed and homogenized tissue with $1 \mathrm{X}$ SDS-loading buffer [48]. Samples were quantified (BioRad RC DC protein assay kit) and normalized prior to electrophoresis by SDS-PAGE. Western blots were conducted as described above. Protein levels were determined by averaging mean pixel intensities measured with Un-Scan-It software (Silk Scientific, Orem, Utah) from three independent western blots.

\section{Gene expression analysis by qRT-PCR}

RNA was isolated using the PureLink RNA Mini Kit (Invitrogen) from young Arabidopsis seedlings. For very small tissues more than 200 young plants were used to obtain enough sample. Shoot apex tissues were taken as the very tip of the young shoots and include the apical meristem. The RNA was quantified and $1 \mu \mathrm{g}$ was added to a reverse transcription reaction with SuperScriptIII (Invitrogen). The cDNAs from these reactions were diluted with $100 \mu \mathrm{l}$ of sterile water and added to qPCR reactions as described by the manufacturer (Roche). qPCR reactions consisted of $1 X$ SYBR Green PCR Master Mix (Roche), and $50 \mathrm{nM}$ of each primer. Primers for the Arabidopsis Twinkle gene were $5^{\prime}$-TCCCCAGAGTCCC AACTCCTGTTGA-3 ${ }^{\prime}$ and $5^{\prime}$-TCCCTGTTCCGCCAAT TTACGCC-3'; for DNA polymerase gamma 1 (At3g20540) were 5'-CCTGAATACCGTTCACGTGCC CA- $3^{\prime}$ and $5^{\prime}$-AGCCGCACTTCCCTGAACAGGA- $3^{\prime}$, and for DNA polymerase gamma 2 (At1g50840) were $5^{\prime}$ TTCCGGCGTCAAAGTCACGTGC-3' and $5^{\prime}$-TGCACT TCCCTGGACTGGAGTGT-3'. Reactions were carried out in a LightCycler 480 System (Roche) for 45 cycles $\left(95^{\circ} \mathrm{C}\right.$ for $10 \mathrm{sec}, 58^{\circ} \mathrm{C}$ for $10 \mathrm{sec}, 72^{\circ} \mathrm{C}$ for $\left.20 \mathrm{sec}\right)$ after initial $5 \mathrm{~min}$ incubation at $95^{\circ} \mathrm{C}$. The fold changes in gene expression were calculated using the $\Delta \Delta C \mathrm{Ct}$ method [49], with the Tub 4 tubulin gene (At5g44340) as an internal control. 


\section{Phylogenetic analysis}

Protein sequences for Twinkle homologues were downloaded from Gen Bank with the following accession numbers: Homo sapiens (NP_068602.2), Caenorhabditis elegans (F46G11.1), Drosophila melanogaster (NP 609318.1), Plasmodium falciparum (NP_702000.1), T7 gp4 (P03692.1), Mus musculus (AAL27647.1), Canis lupus familiaris (XP_543974.1), Arabidopsis thaliana (ACI49800.1), Glycine max (XP_003546288.1), and Oryza sativa Japonica group (BAD46002.1). Multiple sequence alignment was performed using MUSCLE [50] and the evolutionary history was inferred by using the Maximum Likelihood method based on the JTT matrix-based model [51]. The tree with the highest log likelihood $(-3556.6701)$ is shown. Initial trees for the heuristic search were obtained automatically as follows. When the number of common sites was $<100$ or less than one fourth of the total number of sites, the maximum parsimony method was used; otherwise BIONJ method with MCL distance matrix was used. The tree is drawn to scale, with branch lengths measured as the number of substitutions per site. The analysis involved 10 amino acid-coding sequences. The coding data was translated assuming a standard genetic code table. All positions with less than $95 \%$ site coverage were eliminated. That is, fewer than $5 \%$ alignment gaps, missing data, and ambiguous bases were allowed at any position. There were a total of 199 positions in the final dataset. Evolutionary analyses were conducted in MEGA 5 [50].

\section{Abbreviations \\ qRT-PCR: quantitative reverse-transcriptase PCR; mtDNA: mitochondrial DNA; ctDNA: chloroplast DNA; Twinkle: T7 gp4-like protein with intramitochondrial nucleoid localization.}

\section{Competing interest}

The authors declare that they have no competing interests.

\section{Authors' contributions}

JDA performed the tissue-specific western blot and phylogenetic analyses and helped write the manuscript. BL helped conceive the project and made the recombinant protein construct and purified the protein, and performed some of the preliminary assays. JDC performed the GRT-PCR and western blot time course analyses. TH performed the DNA and amino acid sequence analyses. BLN helped conceive the project, performed the primase and helicase assays, and wrote the manuscript with JDA. All authors read and approved the manuscript.

\section{Acknowledgements}

We thank Elisabeth Haws, Tatiana Alarcon, Cynthia Perry and Kali Tibbitts for assistance with some of the experiments. This research was supported in part by a grant from the NIH and by the BYU Mentoring Environments Grant Program.

Received: 18 December 2012 Accepted: 26 February 2013 Published: 4 March 2013

\section{References}

1. Kornberg A, Baker T: DNA Replication, vol 2. New York, New York: W.H. Freeman and Co.; 1991

2. Lionnet T, Spiering MM, Benkovic SJ, Bensimon D, Croquette V: Real-time observation of bacteriophage T4 gp41 helicase reveals an unwinding mechanism. Proc Natl Acad Sci USA 2007, 104(50):19790-19795.
3. Tougu K, Peng H, Marians KJ: Identification of a domain of Escherichia coli primase required for functional interaction with the DnaB helicase at the replication fork. J Biol Chem 1994, 269(6):4675-4682.

4. Iyina TV, Gorbalenya AE, Koonin EV: Organization and evolution of bacterial and bacteriophage primase-helicase systems. J Mol Evol 1992, 34(4):351-357.

5. Shutt TE, Gray MW: Twinkle, the mitochondrial replicative DNA helicase, is widespread in the eukaryotic radiation and may also be the mitochondrial DNA primase in most eukaryotes. $J$ Mol Evol 2006, 62(5): 588-599.

6. Spelbrink JN, Li FY, Tiranti V, Nikali K, Yuan QP, Tariq M, Wanrooij S, Garrido N, Comi G, Morandi L, Santoro L, Toscano A, Fabrizi GM, Somer H, Croxen R, Beeson D, Poulton J, Suomalainen A, Jacobs HT, Zeviani M, Larsson C: Human mitochondrial DNA deletions associated with mutations in the gene encoding Twinkle, a phage T7 gene 4-like protein localized in mitochondria. Nat Genet 2001, 28(3):223.

7. Bernstein JA, Richardson CC: A 7-kDa region of the bacteriophage T7 gene 4 protein is required for primase but not for helicase activity. Proc Natl Acad Sci USA 1988, 85(2):396-400.

8. Patel SS, Picha KM: Structure and function of hexameric helicases 1. Ann Rev Bioc 2000, 69(1):651-697.

9. Korhonen JA, Gaspari M, Falkenberg M: TWINKLE Has 5' -> 3' DNA helicase activity and is specifically stimulated by mitochondrial single-stranded DNA-binding protein. J Biol Chem 2003, 278(49):48627-48632.

10. Seow F, Sato S, Janssen CS, Riehle MO, Mukhopadhyay A, Phillips RS, Wilson RJ, Barrett MP: The plastidic DNA replication enzyme complex of Plasmodium falciparum. Mol Bioc Para 2005, 141(2):145-153.

11. Leipe DD, Aravind L, Grishin NV, Koonin EV: The bacterial replicative helicase DnaB evolved from a RecA duplication. Gen Res 2000, 10(1):5-16.

12. Emanuelsson $\mathrm{O}$, Nielsen $\mathrm{H}$, von Heijne G: ChloroP, a neural network-based method for predicting chloroplast transit peptides and their cleavage sites. Prot Sci 1999, 8(5):978-984.

13. Majeran W, Friso G, Asakura Y, Qu X, Huang M, Ponnala L, Watkins KP, Barkan A, van Wijk KJ: Nucleoid-Enriched Proteomes in Developing Plastids and Chloroplasts from Maize Leaves: A New Conceptual Framework for Nucleoid Functions. Plant Physiology 2012, 158(1):156-189.

14. Wanrooij S, Luoma P, van Goethem G, van Broeckhoven C, Suomalainen A, Spelbrink JN: Twinkle and POLG defects enhance age, dependent accumulation of mutations in the control region of mtDNA. Nucleic Acids Res 2004, 32(10):3053-3064.

15. Tyynismaa H, Sembongi H, Bokori-Brown M, Granycome C, Ashley N, Poulton J, Jalanko A, Spelbrink JN, Holt IJ, Suomalainen A: Twinkle helicase is essential for mtDNA maintenance and regulates mtDNA copy number. Human Mol Gen 2004, 13(24):3219-3227.

16. Tyynismaa H, Mjosund KP, Wanrooij S, Lappalainen I, Ylikallio E, Jalanko A, Spelbrink JN, Paetau A, Suomalainen A: Mutant mitochondrial helicase Twinkle causes multiple mtDNA deletions and a late-onset mitochondrial disease in mice. Proc Natl Acad Sci USA 2005, 102:17687-17692.

17. Sarzi E, Goffart S, Serre V, Chretien D, Slama A, Munnich A, Spelbrink JN, Rotig A: Twinkle helicase (PEO1) gene mutation causes mitochondrial DNA depletion. Ann Neurol 2007, 62(6):579-587.

18. Inoue T, Ide T, Tyynismaa H, Yoshida M, Ando M, Tanaka A, Todaka K, Kang D, Suomalainen A, Sunagawa K: Overexpression of mitochondria DNA helicase, Twinkle, ameliorates cardiac remodeling and failure in mice. Circulation Res 2008, 118(18):S314-S315.

19. Kusakabe T, Richardson CC: Gene 4 DNA primase of bacteriophage T7 mediates the annealing and extension of ribo-oligonucleotides at primase recognition sites. J Biol Chem 1997, 272(19):12446-12453.

20. Kusakabe T, Richardson CC: The role of the zinc motif in sequence recognition by DNA primases. J Biol Chem 1996, 271(32):19563-19570.

21. Kusakabe T, Hine AV, Hyberts SG, Richardson CC: The Cys4 zinc finger of bacteriophage T7 primase in sequence-specific single-stranded DNA recognition. Proc Natl Acad Sci USA 1999, 96(8):4295-4300.

22. Laquel $P$, Litvak $S$, Castroviejo M: Wheat DNA primase: (RNA primer synthesis in vitro, structural studies by photochemical cross-linking, and modulation of primase activity by DNA polymerases). Plant Physiol 1994, 105:69-79.

23. Manchekar M, Scissum-Gunn K, Song D, Khazi F, McLean SL, Nielsen BL: DNA recombination activity in soybean mitochondria. J Mol Biol 2006, 356(2):288-299. 
24. Parent JS, Lepage E, Brisson N: Divergent Roles for the Two Poll-Like Organelle DNA Polymerases of Arabidopsis. Plant Physiology 2011, 156(1):254-262.

25. Cupp JD, Nielsen BL: Arabidopsis thaliana organellar DNA polymerase IB mutants exhibit reduced mtDNA levels with a decrease in mitochondrial area density. Physiol Plantarum 2012. in press, 10.1111/ppl.12009.

26. Tyynismaa $\mathrm{H}$, Suomalainen A: Mouse models of mitochondrial DNA defects and their relevance for human disease. EMBO Rep 2009, 10(2):137-143.

27. Carrie C, Kühn K, Murcha MW, Duncan O, Small ID, OToole N, Whelan J: Approaches to defining dual-targeted proteins in Arabidopsis. Plant $J$ 2009, 57(6):1128-1139.

28. Christensen A, Lyznik A, Mohammed S, Elowsky CG, Elo A, Yule R, Mackenzie SA: Dual-domain, dual-targeting organellar protein presequences in Arabidopsis can use non-AUG start codons. Plant Cell 2005, 17:2805-2816.

29. Mackenzie SA: Plant organellar protein targeting: a traffic plan still under construction. Trends Cell Biol 2005, 15:548-554.

30. Clayton D: Mitochondrial DNA Replication: What We Know. IUBMB Life 2003, 55(4-5):213-217.

31. Manchekar M, Scissum-Gunn KD, Hammett LA, Backert S, Nielsen BL: Mitochondrial DNA recombination in Brassica campestris. Plant Sci 2009, 177(6):629-635.

32. Backert $\mathrm{S}$, Nielsen $\mathrm{BL}$, Borner $\mathrm{T}$ : The mystery of the rings: structure and replication of mitochondrial genomes from higher plants. Trends Plant Sci 1997, 2(12):477-483.

33. Backert S, Borner T: Phage T4-like intermediates of DNA replication and recombination in the mitochondria of the higher plant Chenopodium album (L.). Current Gen 2000, 37:304-314.

34. Oldenburg DJ, Bendich AJ: Mitochondrial DNA from the liverwort Marchantia polymorpha: circularly permuted linear molecules, head-to-tail concatemers, and a 5' protein. J Mol Biol 2001, 310(3):549-562.

35. Wong TW, Clayton DA: Isolation and characterization of a DNA primase from human mitochondria. J Bio/ Chem 1985, 260(21):11530-11535.

36. Hines J, Ray DS: A mitochondrial DNA primase is essential for cell growth and kinetoplast DNA replication in Trypanosoma brucei. Mol Cell Biol 2010, 30:1319-1328.

37. Murthy V, Pasupathy K: Characterization of mitochondrial DNA primase from Saccharomyces cerevisiae. J Biosciences 1994, 19:1-8.

38. Holt IJ, Lorimer HE, Jacobs HT: Coupled leading- and lagging-strand synthesis of mammalian mitochondrial DNA. Cell 2000, 100(5):515-524

39. Bogenhagen DF, Clayton DA: Concluding remarks: The mitochondrial DNA replication bubble has not burst. Trends Bioc Sci 2003, 28(8):404-405.

40. Wanrooij S, Fuste JM, Farge G, Shi Y, Gustafsson CM, Falkenberg M: Human mitochondrial RNA polymerase primes lagging-strand DNA synthesis in vitro. Proc Natl Acad Sci USA 2008, 105(32):11122-11127.

41. Fuste JM, Wanrooij S, Jemt E, Granycome CE, Cluett TJ, Shi Y, Atanassova N, Holt IJ, Gustafsson CM, Falkenberg M: Mitochondrial RNA polymerase is needed for activation of the origin of light-strand DNA replication. $\mathrm{Mol}$ Cell 2010, 37(1):67-78.

42. Nielsen BL, Rajasekhar VK, Tewari KK: Pea chloroplast DNA primase: characterization and role in initiation of replication. Plant Mol Biol 1991, 16:1019-1034.

43. Tuteja N, Phan TN, Tewari KK: Purification and characterization of a DNA helicase from Pea chloroplast that translocates in the 3/-to-5/ direction. Eur J Bioc 1996, 238(1):54-63.

44. Ono Y, Sakai A, Takechi K, Takio S, Takusagawa M, Takano H: NtPoll-like1 and NtPoll-like2, bacterial DNA polymerase I homologues isolated from BY-2 cultured tobacco cells, encode DNA polymerases engaged in DNA replication in both plastids and mitochondria. Plant Cell Physiol 2007, 48:1679-1692

45. Guo S, Tabor S, Richardson CC: The linker region between the helicase and primase domains of the bacteriophage T7 gene 4 protein is critical for hexamer formation. J Biol Chem 1999, 274(42):30303-30309.

46. Claros MG: MitoProt, a Macintosh Application for studying mitochondrial proteins. Comput Appl Biosci 1995, 11:441-447.

47. Song D: Homologous strand exchange and DNA helicase activities in plant mitochondria. Provo: Brigham Young University; 2005.

48. Weigel D, Glazebook J: Arabidopsis: a laboratory manual. Cold Spring Harbor, New York, USA: Cold Spring Harbor Laboratory Press; 2002.

49. Livak K, Schmittgen T: Analysis of relative gene expression data using real-time quantitative PCR and the 2 -(-delta delta $C(T)$ method. Methods 2001, 25:402-408.
50. Tamura K, Peterson D, Peterson N, Stecher G, Nei M, Kumar S: MEGA5: Molecular Evolutionary Genetics Analysis using Maximum Likelihood. Mol Biol Evol: Evolutionary Distance and Maximum Parsimony Methods; 2011.

51. Jones D, Taylor R, Thornton J: The rapid generation of mutation data matrices from protein sequences. Comp App Bios 1992, 8:275-282.

doi:10.1186/1471-2229-13-36

Cite this article as: Diray-Arce et al:: The Arabidopsis At1g30680 gene encodes a homologue to the phage T7 gp4 protein that has both DNA primase and DNA helicase activities. BMC Plant Biology 2013 13:36.

\section{Submit your next manuscript to BioMed Central and take full advantage of:}

- Convenient online submission

- Thorough peer review

- No space constraints or color figure charges

- Immediate publication on acceptance

- Inclusion in PubMed, CAS, Scopus and Google Scholar

- Research which is freely available for redistribution

Submit your manuscript at www.biomedcentral.com/submit
C) Biomed Central 